\title{
Therapeutics
}

\section{Review: in obsessive-compulsive disorder, clomipramine may be more effective than selective serotonin reuptake inhibitors after controlling for other factors}

\author{
Ackerman D, Greenland S. Multivariate meta-analysis of controlled drug studies for obsessive-compulsive disorder. Journal \\ of Clinical Psychopharmacology 2002 June; 22: 309-17. \\ QUESTION: Which characteristics of published trials might account for differences in \\ the effect of clomipramine and selective serotonin reuptake inhibitors in \\ obsessive-compulsive disorder?
}

\section{Data sources}

Studies were identified using Medline, Current Contents, PsychInfo, published reviews and previous meta-analyses.

\section{Study selection}

Studies were eligible if they were randomised, doubleblind parallel trials of at least 8 weeks duration; used the Yale-Brown Obsessive-Compulsive Scale (Y-BOCS) to assess the efficacy of clomipramine or selective serotonin reuptake inhibitors for obsessive-compulsive disorder, and included point estimates, standard deviations or calculable data. The authors included 25 trials.

\section{Data extraction}

Data were extracted on effect size, drug interactions, participant characteristics, inclusion and exclusion criteria, length of trial, trial size and publication year. Metaregression was used to identify sources of heterogeneity.

\section{Main results}

Factors associated with improved response to clomipramine were younger age, early onset obsessivecompulsive disorder, earlier publication date and shorter study duration. Clomipramine was more effective than selective serotonin reuptake inhibitors in placebo trials. This superiority remained after controlling for sources of heterogeneity.

\section{Conclusions}

Although clomipramine has been found to be more effective than selective serotonin reuptake inhibitors in placebo controlled trials, this does not always hold in direct comparisons. This meta-analysis suggests that clomipramine remains effective once sources of heterogeneity are controlled for.

\section{COMMENTARY}

It is unclear whether clomipramine or selective serotonin reuptake inhibitors are most effective for obsessive-compulsive disorder. This meta-analysis reviews knowledge to date, but does not resolve the matter. Although clomipramine performs better than selective serotonin reuptake inhibitors in separate trials, they are equally effective when tested head to head. Non-responders to both classes of medication had more severe cases of obsessive-compulsive disorder. The authors suggest that further analysis of the characteristics of responders and non-responders is needed. Our consortium compared treatment responders and non-responders at 8 international sites. Responders had a higher rate of sudden onset illness, more comorbid impulsive-aggressive disorders and a greater family history of tics. ${ }^{1}$ In contrast, non-responders tended to have more severe disease, poorer insight and more comorbid bipolar and eating disorders. There were no differences in age, gender, age at onset, length of illness or prominent symptom subtypes.

Ackerman et al used multiple regression to account for methodological differences in the studies reviewed. There are several limitations, however.

1) Drug doses were not used in the regression. Some studies used fixed and others flexible doses. Which may have affected efficacy.

2) The studies were published over a 10 year period. During this time there was increasing awareness of the disorder. People with less severe illness were included in later studies. People with less baseline severity may have had a less response to medication, limiting the success of the drug. Later studies may also have included larger numbers of treatment refractory patients.

3) Over time, more people may have been exposed to cognitive behaviour therapy Delayed response to cognitive behaviour therapy may contribute to rising placebo response rates in later trials.

4) Studies of 13 weeks duration may lead to a greater decrease in Y-BOCS scores compared to studies 8 weeks long.

5) The pre-study period varied. An extra pre-study week may have allowed researchers to recognise and discontinue those with significant placebo response or regression to the mean effects.

6) Efficacy was based on percentage change in Y-BOCS. It would be useful to know the extent to which selective serotonin reuptake inhibitors and clomipramine induced symptom remission. A post hoc standardised remission endpoint on the Y-BOCS scale may have great clinical utility.

Future studies of the relative effectiveness of treatments might also 1) use remission as a clinical endpoint, 2) study broader quality of life measures in addition to symptom severity and 3) continue to search for clinical predictors of treatment response. A comparison with other SNRIs may also be useful. Recent studies suggest venlafaxine may be superior to placebo and as effective as clomipramine..$^{2-3}$ Open label trials also suggest that venlafaxine may be effective for treatment resistant obsessive-compulsive disorder. ${ }^{4}$

Professor Eric Hollander and Jessica Kahn Department of Psychiatry, Mt Sinai School of Medicine New York, USA

Hollander, Beinstock CA, Koran LM et al. Refractory Obsessive-Compulsive Disorder: State-of-the-Art Treatment. J Clin Psychiatry 2002;60:20-9.

Ravizza L, Albert U, Ceregato A, et al. Venlafaxine in obsessive compulsive disorder. Fifth International Obsessive Compulsive Disorder Conference; March 29-April 12001 ; Sardinia Italy. Yaryura-Tobias JA, Neziroglu FA. Venlafaxine in Obsessive Compulsive Disorder. Arch Gen Psychiatry 1996;53:653-5. 4

4 Hollander E et al. Venlafaxine in Treatment-Resistant Obsessive Compulsive Disorder, in review. 